\title{
Sparse Wavefield Reconstruction and Source Detection Using Compressed Sensing
}

\author{
Olivier Mesnil ${ }^{1}$, Massimo Ruzzene $^{1,2}$
}

\begin{abstract}
The paper presents a compressed sensing technique for the reconstruction of guided wavefields. Structural inspections based on the analysis of guided wavefields have proven to be effective at detecting and characterizing damage. However, wavefield detection is often a time consuming process, which limits practicality. The proposed reconstruction technique estimates the location of sources and structural features interacting with the waves from a set of sparse measurements. Such features include damage, described as a scattering source. The wavefield is reconstructed by employing information on the dispersion properties of the medium under consideration. The procedure is illustrated through a one-dimensional analytical example, and subsequently applied to the reconstruction of an experimental wavefield in a composite panel with an artificial delamination. The results confirm the ability of the technique to identify the defect, while reconstructing the wavefield with good accuracy using a significantly reduced number of measurements.

Keywords: Lamb wave, Sparse Wavefield Measurements, Compressed Sensing, SHM, NDE, Wavefield Reconstruction

\footnotetext{
Email address: omesnil3@gatech.edu (Olivier Mesnil)

${ }^{1}$ D. Guggenheim School of Aerospace Engineering, Georgia Institute of Technology, Atlanta, GA, 30332, US

${ }^{2}$ G. W. Woodruff School of Mechanical Engineering, Georgia Institute of Technology, Atlanta, GA, 30332, US
} 


\section{Introduction}

Lamb wave-based inspection continues to draw significant attention within the NonDestructive Evaluation (NDE) and Structural Health Monitoring (SHM) communities [1]. A first group of techniques employs arrays of transducers mounted on the structure at a predetermined set of points $[2,3,4,5,6]$. These techniques generally provide a fast estimate of the location of defects such as holes, cracks, or delaminations, but often provide limited information on their shape, size and thickness-wise location. A second group of techniques relies on wavefield detection and analysis $[7,8,9,10]$. In this context, a wavefield denotes a series of images describing the time or frequency evolution of a propagating wave. Wavefield techniques provide a wealth of information that can effectively locate as well as quantify damage $[7,11]$. However, wavefield detection is a timely process due the common need for multiple averages at each location to mitigate potentially low signal-to-noise ratios, and the large number of measurements required to avoid spatial aliasing and resolve the desired information. Therefore, there is a recognized need to reduce acquisition time by reducing the number of acquisitions. This is the focus of recent papers where the use of dedicated equipment is explored to speed up acquisitions by increasing the number of measurements per unit of time. Examples include the use of a multi-point Laser Doppler Vibrometer (LDV) [12, 13], and of a galvanometer mirror system in conjunction with a high amplitude single frequency piezoelectric transducer [14]. This paper contributes to this objective by exploring a process that reduces the number of required measurements, possibly below the limits imposed by Nyquist sampling theorem. The proposed process is based on the hypothesis that the time response recorded at one point of the surface of a plate structure can be employed to infer the response at any other point assuming prior knowledge of dispersion relations, plate geometry, and location of the excitation. When this knowledge is incomplete due to the presence of defects for example, this extrapolation is not straightforward and tools such as Compressed 
Sensing $[15,16]$ must be used. Compressed Sensing (CS) is a mathematical theory commonly used as a reconstruction process for data sampled below the Nyquist frequency. Compressed sensing deals with "sparse" or "compressible" signals randomly sampled within the domain of interest, either time or space. A "sparse" signal is here intended as a signal with only a few nonzero coefficients, whereas a signal is "compressible" if there exist a basis in which the signal has a sparse representation. Applications of CS include image processing [17] and magnetic resonance imaging [18]. Prior applications of CS to the reduction of wavefield measurements can be found in [19], where, in contrast to the present paper, no prior knowledge of the physical properties of the medium such as its dispersion relations is assumed. A more recent paper from the same group [20] presents a wavefield reconstruction technique similar to the one presented herein, but using analytical dictionaries such as wavelets, Fourier functions or Gabor atoms as bases for wavefield reconstruction. Another series of papers develops a technique called sparse "wavenumber analysis" [21, 22] for reconstructing the dispersion relations of a plate through sparse measurements. This technique has also been recently applied to pristine wavefield reconstruction [23]. The present paper presents a process to reconstruct a wavefield on an oversampled grid from few measurements. The paper differentiates itself from prior work by performing the reconstruction upon locating the non-pristine material points interacting with the wavefronts. The estimated dispersion relations of the medium are then used to extrapolate the wavefield onto a grid of points of arbitrary size and density. The estimated dispersion relations form the basis that sparsifies the wavefield, and allow wavefield reconstruction. Furthermore, this basis locates all features within the plate that interact or affect the wavefield. These include sources or scatterers such as damage. The number of measurements required for the reconstruction is significantly smaller than the number of measurements required by the common sampling requirements.

The paper is organized as follows. Following this introduction, Section 2 provides a sum- 
mary of the CS formulation necessary for the development of the process. Section 3 contains the details of the matrix formulation and of the wavefield reconstruction process. Next, Section 4 illustrates the application of the reconstruction process to a one-dimensional (1D) analytically generated wavefield with a reflector, and presents results on a two-dimensional (2D) experimental wavefield on a composite panel containing an artificial delamination. Finally, Section 5 summarizes the main findings of the work and provides recommendation for future investigations.

\section{Overview of Compressed Sensing}

The fundamental mathematical result of Compressed Sensing (CS) states that if a signal $\boldsymbol{x} \in \mathbb{R}^{N \times 1}$ is $K$ sparse, i.e. only $K$ components of $x$ are non zero, it can be exactly reconstructed with an overwhelming probability from few linear measurements randomly chosen $[15,16]$. The same result holds for compressible signals, which are signals well approximated by a small number of coefficients in a given basis. The basis in which a compressible signal is sparse is called a "sparsifying basis". In here and in the remainder of the paper, bold lower case letters denote vectors, while capitalized bold letters will be use for matrices.

The general under-sampling problem can be expressed as :

$$
\boldsymbol{y}=\boldsymbol{\Phi} \boldsymbol{x}
$$

where $\boldsymbol{y} \in \mathbb{R}^{M \times 1}$ is the measurement vector, $\boldsymbol{\Phi} \in \mathbb{R}^{M \times N}$ is the down-sampling measurement matrix and $\boldsymbol{x} \in \mathbb{R}^{N \times 1}$ is the unknown vector to reconstruct. It is assumed that $M<N$, so that $\boldsymbol{\Phi}$ is a "short and large" type of matrix. In other words, the number of linear measurements is smaller than the number of unknown variables in $\boldsymbol{x}$.

For a compressible signal, the problem can be rewritten through a change of basis as 
follows:

$$
y=\Phi B \alpha=A \alpha
$$

where $\boldsymbol{\alpha} \in \mathbb{R}^{P \times 1}$ is the sparse representation of $\boldsymbol{x}$ in the basis defined by $\boldsymbol{B} \in \mathbb{R}^{N \times P}$, i.e. $\boldsymbol{A}=\boldsymbol{\Phi} \boldsymbol{B}$, where $\boldsymbol{A} \in \mathbb{R}^{M \times P}$ is the denoted as the "sensing matrix". Thus:

$$
\boldsymbol{x}=\boldsymbol{B} \boldsymbol{\alpha}
$$

with $P$ denoting the number of vectors forming the basis $\boldsymbol{B}$. Equation (2) defines a CS problem. The objective is the estimation of the basis coefficients $\boldsymbol{\alpha}$ that provide the best reconstruction of the unknown set of physical variables $\boldsymbol{x}$ represented through $\boldsymbol{B}$. Inputs to the problem are the measurements $\boldsymbol{y}$. Several algorithms have been proposed for the solution of CS problems as in Equation (2). For example, greedy algorithms such as the Orthogonal Matching Pursuit is proposed in [24, 25]. As an alternative, $l 1$-minimization algorithms such as Basis Pursuit have shown suitability for the reconstruction of noisy sparse signals [26, 27]. Finally, Total Variation (TV) algorithms have been employed when the gradient of the signal to reconstruct is sparse $[28,29]$. An $l 1$-minimizer is selected for the solution of the CS problem in this paper due to its robustness in the presence of measurement noise. The application of l1-minimizers requires that the sensing matrix $\boldsymbol{A}$ verifies the Restricted Isometry Property (RIP) with a RIP constant $\delta_{s}$ smaller than unity [30]. This constant is defined for a matrix $\boldsymbol{A}$ by the smallest scalar verifying the following inequality for all $\boldsymbol{y}$ and for all sub-matrices of $\boldsymbol{A}$, denoted $\boldsymbol{A}_{s}$ :

$$
\left(1-\delta_{s}\right)\|\boldsymbol{y}\|_{2}^{2} \leq\left\|\boldsymbol{A}_{s} \boldsymbol{y}\right\|_{2}^{2} \leq\left(1+\delta_{s}\right)\|\boldsymbol{y}\|_{2}^{2}
$$

The $\delta_{s}$ constant is a characterization of the nearly orthogonal matrices operating on sparse vectors. The case $\delta_{s} \approx 0$ corresponds to a nearly orthonormal matrix, while $\delta_{s} \approx 1$ indicates that some of the vectors forming the matrix $\boldsymbol{A}$ are nearly identical or redundant. This 
requirement guarantees that the CS problem can be inverted with an overwhelming probability through l1-minimization. There exists a list of candidate matrices $\boldsymbol{A}$ obeying this property. These include the random Gaussian, the Bernoulli and the partial Fourier matrices [27]. However, computing these RIP constants is a non-deterministic polynomial-time hard problem and is not possible for most matrices [31]. In practice this requirement is often replaced by ensuring that matrices $\boldsymbol{B}$ and $\boldsymbol{\Phi}$ are incoherent [32,30]. This is mathematically verified by checking that the coherence, i.e. the maximum value of the scalar product between all the columns of the $\boldsymbol{A}$ matrix, is smaller than a constant defined in [33], meaning that the matrix $\boldsymbol{A}$ is nearly orthonormal.

The Basis Pursuit Denoising (BPDN) [26, 27] algorithm is a l1-minimization algorithm that guarantees exact reconstruction [34] by solving the following problem

$$
\min \|\boldsymbol{\alpha}\|_{1} \text { subject to }\|\boldsymbol{y}-\boldsymbol{A} \boldsymbol{\alpha}\|_{2}^{2} \leq \sigma
$$

where $\sigma$ is a constant related to the noise level in the measurements, with $\sigma=0$ in the absence of noise. The BPDN algorithm used is this paper is SGPL1, which is a solver for large scale sparse reconstruction that employs convex optimization to find a sparse representation of $\boldsymbol{\alpha}$ even when $\boldsymbol{B}$ is an over-complete dictionary of basis functions [35, 36]. Accordingly, an estimation of the sparse optimum can be found even if the vectors in $\boldsymbol{B}$ are coherent with one another. As many of the BPDN solvers, SPGL1 is robust to measurement noise and takes $\sigma$ as the only input parameter.

\section{Guided wavefield reconstruction through Compressed Sensing}

We formulate the reconstruction process by considering a wavefield as a compressible signal in space. Accordingly, let $P$ be the number of regularly spaced pixels on which a wavefield is defined. The goal is to reconstruct the wavefield from the knowledge of $M$ sparse 
measurements such that $M<P$. The dispersion relations of the media and the Lamb wave propagation equation are used to formulate a sparsifying basis for the wavefield. In the proposed process, location of the sources are first estimated from $M$ measurements by means of a l1-minimizer and the sparsifying basis (Equation (3)). The estimated sources are then used to extrapolate, or reconstruct, the wavefield over a desired grid of $P$ points, which can be of arbitrary size and density. For the cases presented in the paper, the fully measured wavefield is used to assess the quality of the reconstructed wavefield. All estimations are based on the expression of excitation and response in the frequency domain.

\subsection{CS problem formulation}

The Lamb wave propagation equation [37] for a single propagating mode $(\mu)$ is used to express the time evolution of the response $y$ at point $m,(m=1, . ., M)$ :

$$
y_{m}(t)=\sum_{s=1}^{S} \frac{1}{\sqrt{d_{m, s}}} \mathcal{F}^{-1}\left(\mathcal{F}\left(v_{s}^{(\mu)}(t)\right) e^{-i k_{m, s}^{(\mu)}(f) d_{m, s}}\right)
$$

Here $S$ is the number of sources emitting wavefronts, $d_{m, s}$ is the distance between the source $s$ and the measurement point $m$, while $\mathcal{F}$ and $\mathcal{F}^{-1}$ denote the Fourier transform and its inverse. Furthermore, $v_{s}^{(\mu)}(t)$ is the excitation function of mode $(\mu)$ at source $s, f$ is frequency, and

$k_{m, s}^{(\mu)}(f)$ is the wavenumber of mode $(\mu)$ along the line joining the source $s$ to the measurement point $m$. Equation (4) assumes that amplitude decays solely due to geometrical spreading, and therefore material dissipation is neglected. Equation (4) can be conveniently expressed in the frequency domain:

$$
y_{m}(f)=\sum_{s=1}^{S} \frac{1}{\sqrt{d_{m, s}}}\left(v_{s}^{(\mu)}(f) e^{-i k_{m, s}^{(\mu)}(f) d_{m, s}}\right)
$$

The objective is to reconstruct the wavefield with a limited number of measurements and without prior knowledge of all sources that contribute to the propagation of guided waves 
within the plate-like structure under consideration. Surface mounted transducers, boundaries, structural features such as holes, interfaces, stiffeners, and defects are considered as potential sources. Since their number and location is initially unknown, all points belonging to the pixellated region of interest are treated as potential source locations. Thus, the number of pixels $P=S$ in Equation (5). In general, the grid of potential sources is defined to at least satisfy Nyquist criterion for proper sampling of the wavefield, i.e. two per wavelength.

\subsection{Matrix formulation}

Assuming multi-modal wave propagation, Equation (5) can be expressed in matrix form as follows:

$$
\boldsymbol{y}_{m}(f)=\sum_{\mu=1}^{N^{(\mu)}} \boldsymbol{H}^{(\mu)}(f) \boldsymbol{v}^{(\mu)}(f)
$$

where $\boldsymbol{y}(f) \in \mathbb{R}^{M \times 1}$ is the vector containing the measurements in the frequency domain:

$$
\boldsymbol{y}(f)=\left[y_{1}(f), \cdots, y_{M}(f)\right]^{T}
$$

Also, $N^{(\mu)}$ is the number of propagating modes, while $\boldsymbol{H}^{(\mu)}(f)$ is the matrix of basis functions whose $m, s$ term is:

$$
h_{m, s}^{(\mu)}(f)=\frac{e^{-i k_{m, s}^{(\mu)}(f) d_{m, s}}}{\sqrt{d_{m, s}}}
$$

Furthermore, vector $\boldsymbol{v}^{(\mu)}(f) \in \mathbb{R}^{S \times 1}$ is given by:

$$
\boldsymbol{v}^{(\mu)}(f)=\left[v_{1}^{(\mu)}(f), \cdots, v_{S}^{(\mu)}(f)\right]^{T}
$$

and contains unknown amplitude and phase information for source $s$ at frequency $f$. In this paper, the basis functions are selected upon assumption of the dispersion relations of the structure, which is based on knowledge of thickness and material properties. Specifically, the wavenumbers $k_{m, s}^{(\mu)}(f)$ are estimated using the Semi-Analytical Finite Element method 
(SAFE) described in [38]. Hence, for every measurement-sources pair, the only unknown in Equation $(5)$ is $v_{s}^{(\mu)}(f)$. While it is understood that the assumption of the dispersion properties is a source of uncertainty and potential errors, this choice allows the formulation of the problem within the framework under consideration, while employing knowledge of the physics of wave motion. This is considered a viable approach that guides the choice of the basis function based on physical considerations, and not solely on interpolation principles. However, the robustness of this technique and the uncertainty introduced by this assumption needs to be investigated, and will be the object of future studies.

Here, all investigations and experiments consider frequencies that are below the $A 1$ and $S 1$ mode cut-off frequencies so that only the $A 0$ and $S 0$ modes propagate in the specimen. While this choice is made for convenience of experimentation and computation, it is noted that the formulation can be extended to include a higher number of modes. Accordingly, for $N^{(\mu)}=2$, Equation (6) can be rewritten as:

$$
\boldsymbol{y}(f)=\boldsymbol{H}^{S 0}(f) \boldsymbol{v}^{S 0}(f)+\boldsymbol{H}^{A 0}(f) \boldsymbol{v}^{A 0}(f)=\boldsymbol{H}(f) \boldsymbol{v}(f)
$$

where $\boldsymbol{H}(f)$ and $\boldsymbol{v}(f)$ are obtained respectively by horizontal and vertical concatenations of the corresponding matrices and vectors. The dimensions of $\boldsymbol{H}(f)$ are $M \times S N^{(\mu)}$, where $S$ is the number of potential sources and $M$ the number of measurement.

Equation (8) is in the same form of Equation (2), so that the following CS problem can be solved at a given frequency $f$ :

$$
\min \|\boldsymbol{v}(f)\|_{1} \text { subject to }\|\boldsymbol{y}(f)-\boldsymbol{H}(f) \boldsymbol{v}(f)\|_{2}^{2} \leq \sigma
$$

The 11 -minimization in Equation (9) estimates amplitude and phase of each component of the source vector $\boldsymbol{v}(f)$, and therefore defines the contribution of the potential sources to the measured wavefield. It is worthwhile emphasizing that this source vector includes 
excitation provided by transducers employed to originate the wavefield along with any sources of scattering resulting from both known structural features and unknown defects. Of note is also the fact that the contribution to each mode is evaluated separately, which accounts for the fact that excitation through surface mounted transducers such as piezoelectric disc and scattering occurs with different efficiency for each participating mode. The sparsity of the formulation exploited herein relies on the fact that vector $\boldsymbol{v}(f)$ is sparse in space, which is based on the reasonable assumption that the number of sources, either known or unknown, can be considered small compared to the number of pixels in the region of interest. Therefore only a few components of $\boldsymbol{v}(f)$ are expected to be non-zero. Note that the wavenumbers $k_{m, s}^{(\mu)}(f)$ are functions of direction, hence the dictionary $\boldsymbol{H}(f)$ created with the basis functions $h_{m, s}^{(\mu)}(f)$ shares the isotropy or anisotropy property of the material through the dispersion relations $k_{m, s}^{(\mu)}(f)$. However it is assumed that every source is omni-directional in amplitude and no empirical or theoretical model is used to infer the amplitude directionality of each source because amplitude directionality may come from either the nature of the material or from the nature of the source or scatter.

\subsection{Implementation and wavefield reconstruction}

The wavefield reconstruction process can be described by the two steps process depicted in Figure 1. The first step consists in the estimation of the excitation function spectra for each potential sources and each mode through l1-minimization. The inputs of this step are the $M$ measurements and the sensing matrix $\boldsymbol{H}(f)$, created using the dispersion relations of the media and the location of measurements and potential sources. The measurements are selected from a jittered distribution [39], as depicted in Figure 2, meaning that one measurement is randomly selected within each cell of a regular grid defined in the region of interest. A Compression Ratio (CR) of the reconstructed wavefield with respect to Nyquist theorem is 
defined by:

$$
C R(\%)=100 \times\left(1-\frac{M}{M_{N y q}}\right)
$$

where $M_{N y q}$ is the number of measurements required by Nyquist theorem. This is determined by considering two measurements per minimum wavelength in the considered frequency range of excitation. The $\mathrm{CR}$ expresses the reduction of the number of measurements to acquire with respect to the full capture of the wavefield based on sampling criteria. Critical to the CS problem solution is the choice of the stopping criteria $\sigma$ in Equation (3). In practice, relating $\sigma$ to the norm of the measurement has been proven to be efficient [40] therefore $\sigma=\frac{\|\boldsymbol{y}(f)\|_{2}}{10}$ is fixed.

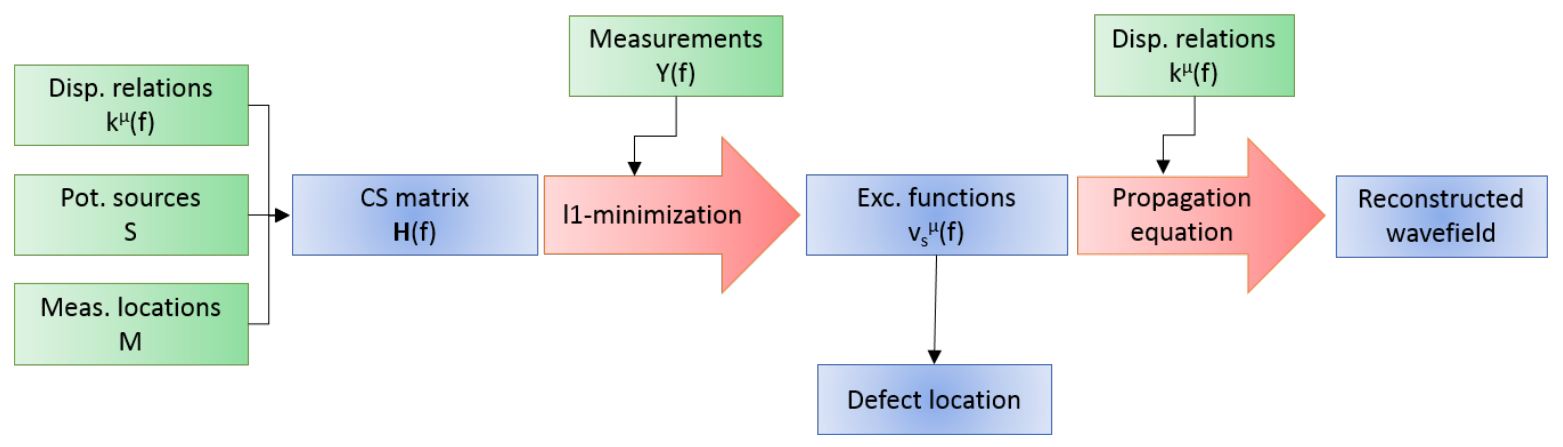

Figure 1: Schematic of the two step wavefield reconstruction process.

The first step provides as an output the vector $\boldsymbol{v}(f)$. This is performed by employing the Basis Pursuit solver SPGL1 [36] which is used to invert Equation (8) through l1-minimization of Equation (3). In practice, looking for a sparse representation of the wavefield in the basis $\boldsymbol{H}(f)$ constrains the wavenumber on a finite set of values for a frequency and direction of propagation. In other words, for a given pair of measurement-source forming an angle $\theta_{0}$ with respect to a reference direction and at frequency $f_{0}$, the wavenumber is constrained to take one of the following two scalar values: $k^{S 0}\left(f_{0}, \theta_{0}\right)$ or $k^{A 0}\left(f_{0}, \theta_{0}\right)$. These values are based on dispersion properties estimated on the undamaged part. Delaminations cause a change in 


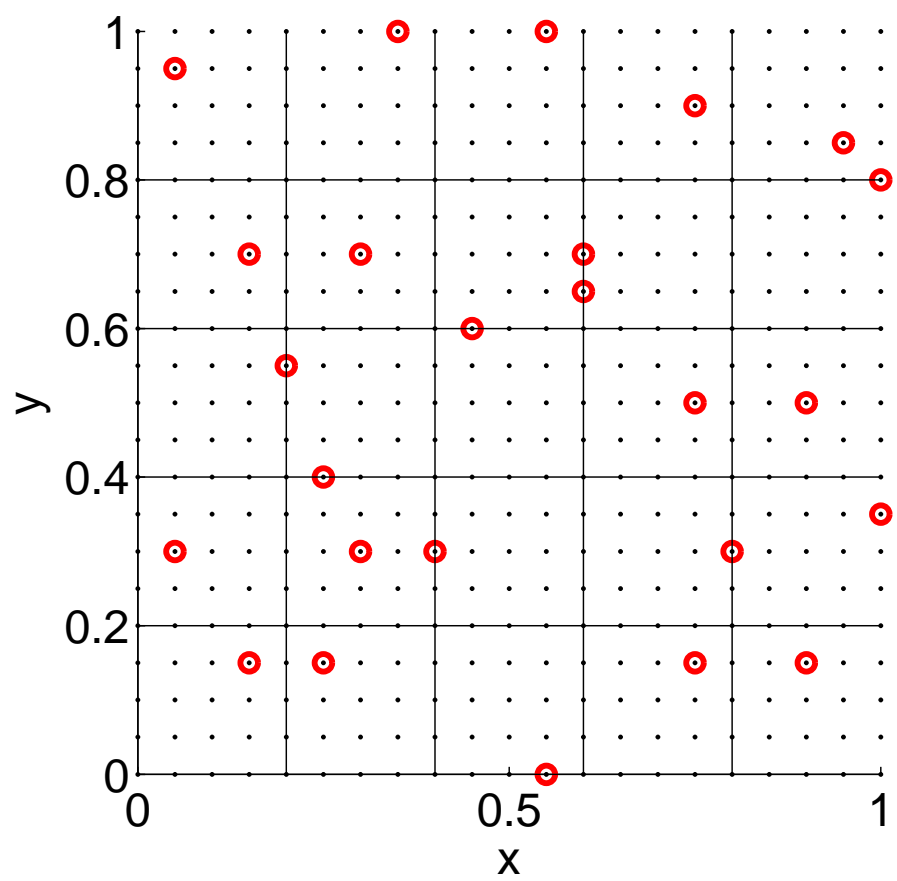

Figure 2: Jittered sampling of the measurement region: the region of interest is split in cells (black lines) and one measurement (red circles) is taken randomly within each cell. The black dots represent the points required for spatial discretization according to Nyquist criterion.

wavenumber as illustrated in $[11,41,42]$. These modified wavenumbers, as defined by damage, are not known a priori, and are therefore not included in the reconstruction bases. Thus, the reconstruction is not accurate within the delaminated region, which is still however identified by a cluster of effective sources. The vector $\boldsymbol{v}(f)$ contains information on the location of the sources as well as on their amplitude and phase for each mode and frequency. As such, $\boldsymbol{v}(f)$ may be used to detect and locate defects. Since the problem is solved at each frequency, and in order to have a visual representation of the location of the sources that are active 
contributors to the wavefield, the following quantity is monitored:

$$
\left|v_{s}^{(\mu)}\right|=\frac{1}{\Delta f} \int_{f} v_{s}^{(\mu)}(f) d f
$$

where $\Delta f$ defines the considered bandwidth selected for the reconstruction. Mapping of the resulting vector $\left|\boldsymbol{v}^{(\mu)}\right|$ over the entire pixellated region provides a map of active sources including defects. The pixels for which vector $\left|\boldsymbol{v}^{(\mu)}\right|$ is greater than zero represent the active sources of mode $(\mu)$ and identifies a feature that contributes to the considered modal component of the wavefield.

The second step consists in the reconstruction of the wavefield, which involves the forward application of Equation (8), by employing the estimated $\boldsymbol{v}(f)$ into the propagation equation (Equation (5)). The wavefield is extrapolated to a desired set of points that may be part of a grid of points of arbitrary size and density. In this paper, the wavefields are only reconstructed on the grid of points of the fully measured wavefield for comparison purposes.

The quality of the reconstruction is evaluated by the magnitude squared coherence defined as

$$
\mathcal{C}(\boldsymbol{r}, f)=\frac{\left|S_{M s, R c}(\boldsymbol{r}, f)\right|^{2}}{S_{M s}(\boldsymbol{r}, f) S_{R c}(\boldsymbol{r}, f)}
$$

where $S_{M s}$ and $S_{R c}$ are the auto-spectral densities of the fully measured and of the reconstructed wavefield respectively, while $S_{M s, R c}(\boldsymbol{r}, f)$ is the cross-spectral density between the two wavefields. The coherence at the excitation frequency $f_{0}$ may be averaged over the spatial extent of the region of interest to evaluate the effect of the number of measurement points $M$ and is denoted $<\mathcal{C}\left(f_{0}\right)>$. Alternatively, the average coherence is estimated as a function of frequency $\langle\mathcal{C}(f)>$ to evaluate the quality of the reconstruction over the excitation bandwidth. Finally, the spatial variation of the coherence at the excitation frequency $\mathcal{C}\left(\boldsymbol{r}, f_{0}\right)$ defines the quality of the reconstruction over the domain of interest. These various metrics are employed in the following examples to illustrate the performance of the reconstruction 
process.

\section{Results}

The reconstruction approach is illustrated first on an analytically generated wavefield, and is then applied to the analysis of experimental results on a composite plate with an artificial delamination.

\subsection{Reconstruction of a one-dimensional analytical wavefield}

A one dimensional (1D) analytically generated wavefield is produced by a point source and affected by a point scatterer (Figure 3). The superposition of two modes propagating at different wave velocities simulates wavefield conditions typically encountered during testing. The propagation equation in Equation (5) is used with wavenumbers corresponding to those of $1.6 \mathrm{~mm}$ a glass fiber composite plate. Propagation is simulated along a $200 \mathrm{~mm}$ long line. The excitation function is a $200 \mathrm{kHz} 4$-cycle sine burst that excites both $A 0$ and $S 0$ modes at the same amplitude. The transducer location is at $x=30 \mathrm{~mm}$, while the scatterer is at $x=180 \mathrm{~mm}$. The scatterer reflects and converts the incident waves as shown in Figure 3 . The bandwidth of analysis, estimated on the basis of the considered excitation, is $100-300$

$\mathrm{kHz}$. The corresponding smallest wavelength is the one of the $A 0$ mode, which at $300 \mathrm{kHz}$ is equal to $4.2 \mathrm{~mm}$. Hence, resolution of the wavefield according to Nyquist theorem requires $M_{N y q}=94$ regularly spaced measurements along the line.

We assume that the location of both transducer and scatterer is to be determined out of a grid of potential sources $S=94$, which defines the dimensions of the unknown vector $\boldsymbol{v}(f)$. This vector comprises of $S \times N^{(\mu)}=188$ components to include the contribution of the two modes $A 0$ and $S 0$. A total of $M=50$ measurements are randomly selected along the length of the considered domain and fed to the $l 1$-minimization solver to complete step one of the 


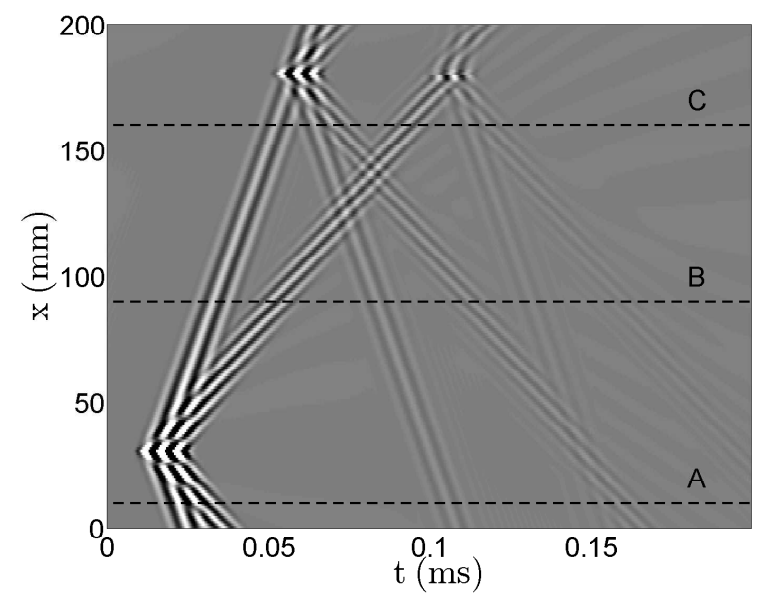

Figure 3: Simulated analytical 1D wavefield showing the propagation, reflection and conversion of two guided wave modes.

process. This corresponds a compression ratio of $C R=47 \%$. The active sources estimated through l1-minimization are visualized by plotting the corresponding excitation amplitude $\left|v_{s}^{S 0}\right|+\left|v_{s}^{A 0}\right|$, defined by Equation (11), as a function of the assumed grid points (Figure 4). The potential sources at which $\left|v_{s}^{S 0}\right|+\left|v_{s}^{A 0}\right| \approx 0$ represent the pristine region of the specimen. Active sources, i.e. the set of potential sources at which $\left|v_{s}^{S 0}\right|+\left|v_{s}^{A 0}\right|>0$, are identified to be exactly located at the location of both the transducer and the reflector, illustrating how the process can be used as a defect detection method.

In the second step of the process, the estimated $\boldsymbol{v}(f)$ is employed in the forward propagation equation to reconstruct the wavefield along the considered 1D spatial domain. The result of the reconstruction process is shown in Figures $5 \mathrm{a}$ and $5 \mathrm{~b}$ for the $S 0$ and the $A 0$ mode respectively, which illustrates how the process allows the separate estimation of the participating modes. The quality of the reconstruction is shown by comparing the actual response with the estimated at the three spatial locations A,B and $\mathrm{C}$ highlighted in Figure 3. The 


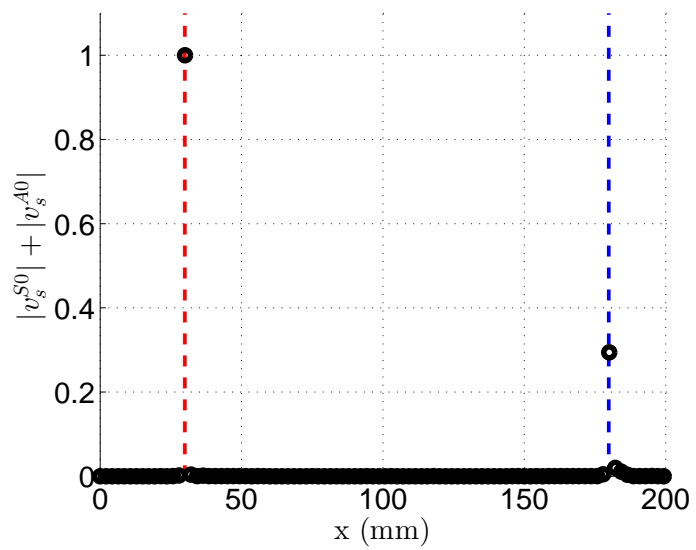

Figure 4: Estimated amplitude of excitation $\left|v_{s}^{S 0}\right|+\left|v_{s}^{A 0}\right|$ along the considered discretization grid with $S=94$ of the 1D spatial domain. The estimates are based on $M=50$ measurements randomly selected along the length of the considered domain. The actual position of the main (red) and secondary (blue) sources are shown by the vertical dashed lines.

comparisons reported in Figure 6 show the accuracy of the reconstruction, whose difference with the actual response is visually negligible. The very small differences are related, in the absence of measurement noise, to the non-zero stopping criteria $\sigma$ of the $l 1$-minimization. We note that several attempts were made to judge the predictability of the minimization process by changing the initial set of measurements and no significant differences were observed between the results. Furthermore, no significant convergence challenges were ever encountered in all cases run. This gives us some confidence that the minimum search finding process is robust and leads to a solution that is physically representative. This is further substantiated by the estimation of correlation values.

The quality of the reconstruction can be better quantified by evaluating the coherence defined in Equation (12). First, the spatially averaged coherence is evaluated as a function of frequency. The estimation for $M=50$ shows how the reconstruction is effective in terms 


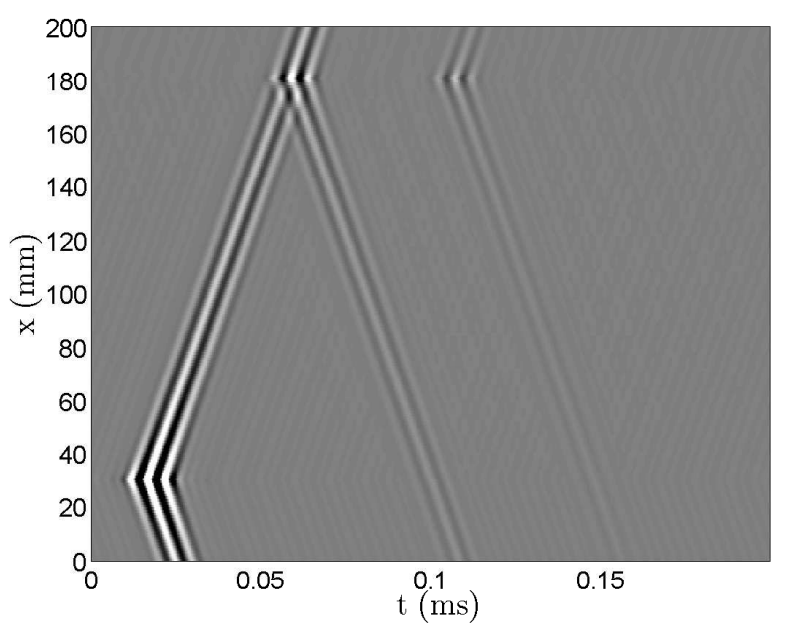

(a)

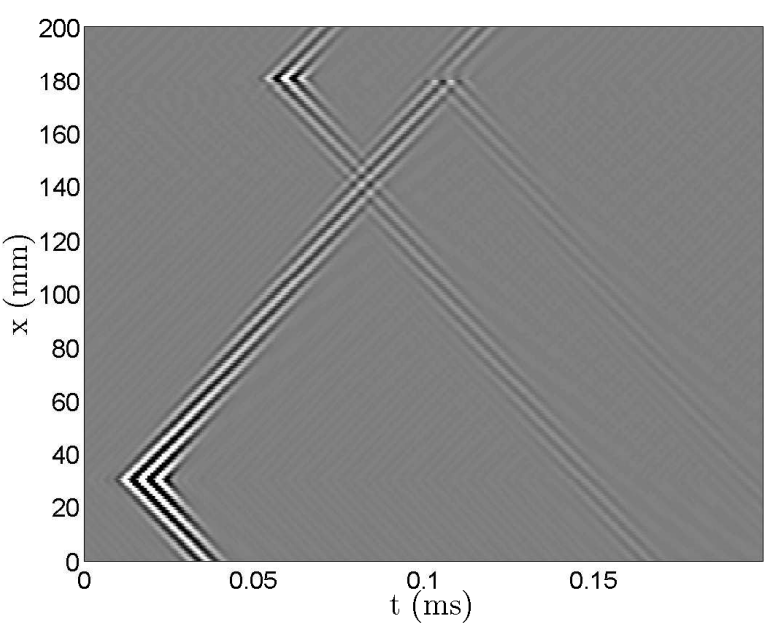

(b)

Figure 5: Reconstructed wavefield obtained using 50 measurements, (a): $S 0$ mode and (b): $A 0$ mode.

of coherence in the relevant frequency range $100-300 \mathrm{kHz}$ (Figure 7a). Next, the spatially averaged coherence is evaluated at the excitation frequency for a different number of measurement points $M$. The results of Figure $7 \mathrm{~b}$ show that coherence values greater than 0.9 are obtained for numbers of measurements as low as $M=18$ corresponding to a compression ratio $C R=82 \%$. In Figure $7 \mathrm{~b}$, the dashed red line is a reference corresponding to $M_{N y q}=94$, the theoretical lower bound imposed by sampling considerations according to Nyquist theorem.

Since the results presented in this section have been created analytically, it is valuable to study the influence of an inexact estimation of the material properties used to generate the dispersion relations of the specimen. To this end, the dispersion relations are scaled by a parameter $\gamma$ using the following relation:

$$
k_{m, s}^{\gamma(\mu)}(f)=(1+\gamma) k_{m, s}^{(\mu)}(f)
$$




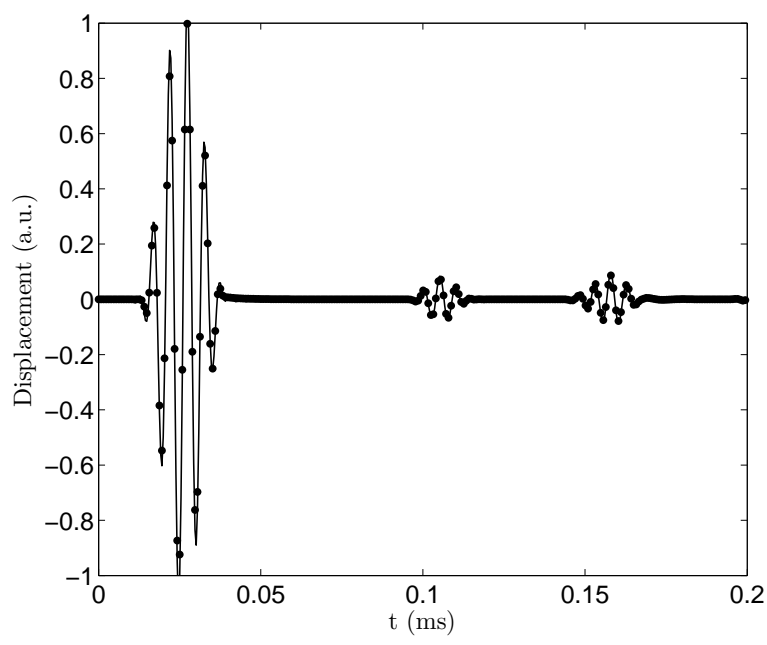

(a)

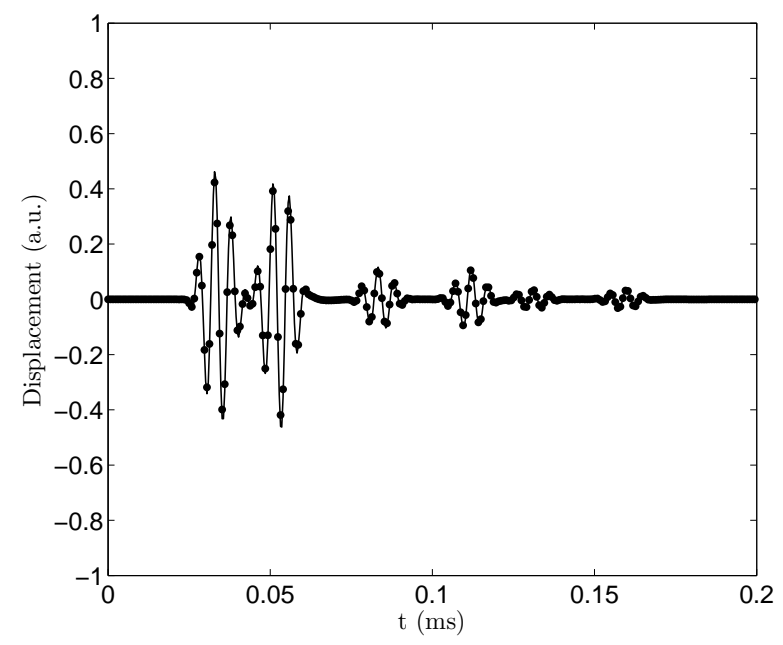

(b)

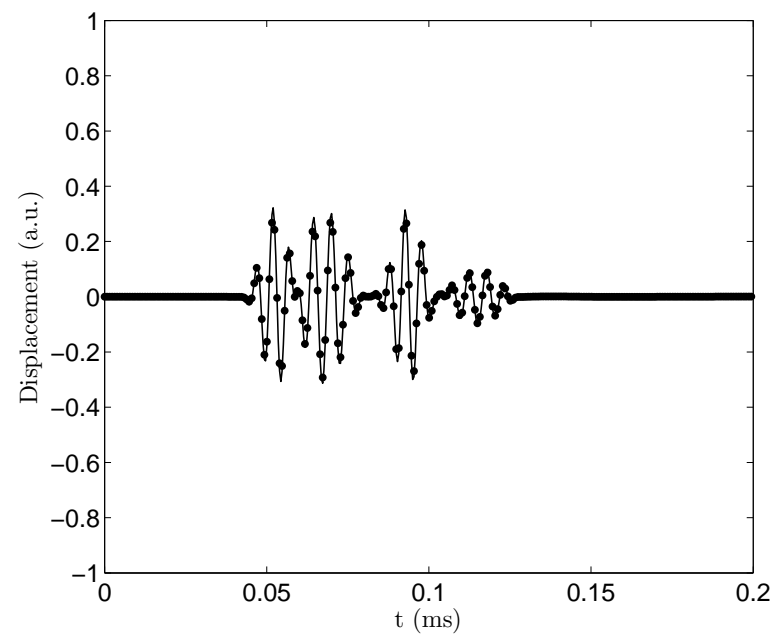

(c)

Figure 6: True measurements (black dots) and reconstructed signals from 50 measurements (black lines) for the selected points (a): A, (b): B and (c): C. 


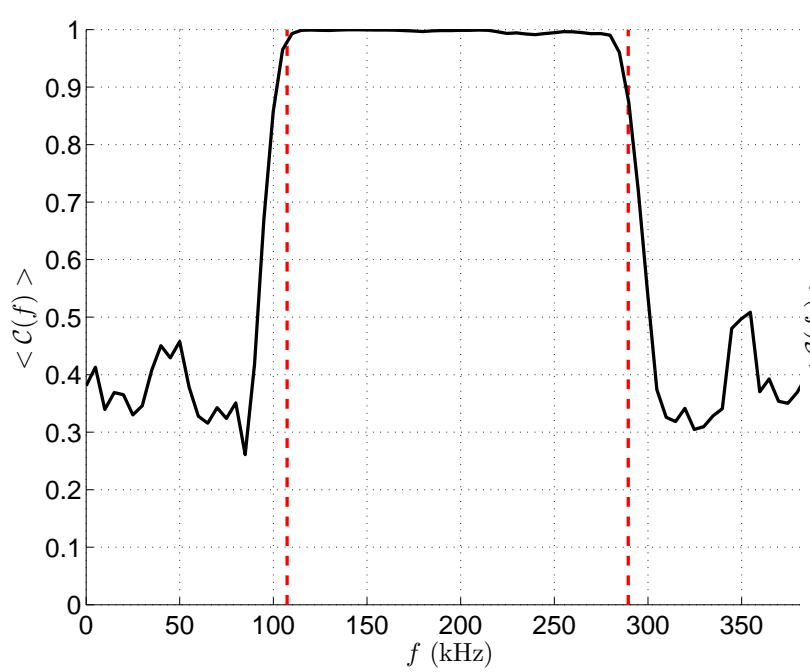

(a)

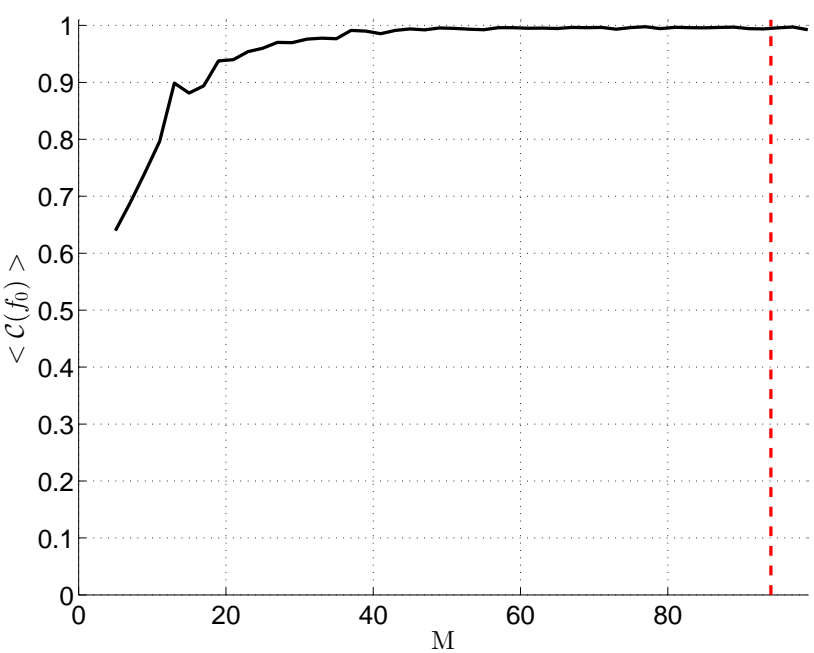

(b)

Figure 7: Spatially averaged coherence of reconstructed wavefield: (a) coherence versus frequency $<\mathcal{C}(f)>$ for $M=50$ and (b): coherence at excitation frequency $<\mathcal{C}\left(f_{0}\right)>\left(f_{0}=200 \mathrm{kHz}\right)$ versus number of measurements.

where $k_{m, s}^{\gamma(\mu)}(f)$ is the wavenumber scaled by the parameter $\gamma$. Even though this alteration of the dispersion relations is trivial and may not accurately represent the conditions encountered in experiment, the purpose of this analysis is to study the influence of inexact estimation of the dispersion relations on the reconstruction quality. Reconstruction is then conducted with the same parameters as previously, i.e. $M=50$ and $S=94$. The coherence of the reconstruction averaged in space at the dominant frequency is then extracted and displayed as a function of $\gamma$ in Figure 8.

According to Figure 8, the reconstruction quality is highly sensitive to the precision of the dispersion relations. More precisely, dispersion relations over or underestimated by $10 \%$ will result in a drop of the reconstruction coherence of about $20 \%$. However it must be noticed that the reconstruction coherence stays above 0.7 even for a miscalculation of the dispersion relations of $50 \%$. In practice, it can be assumed that dispersion relations are 


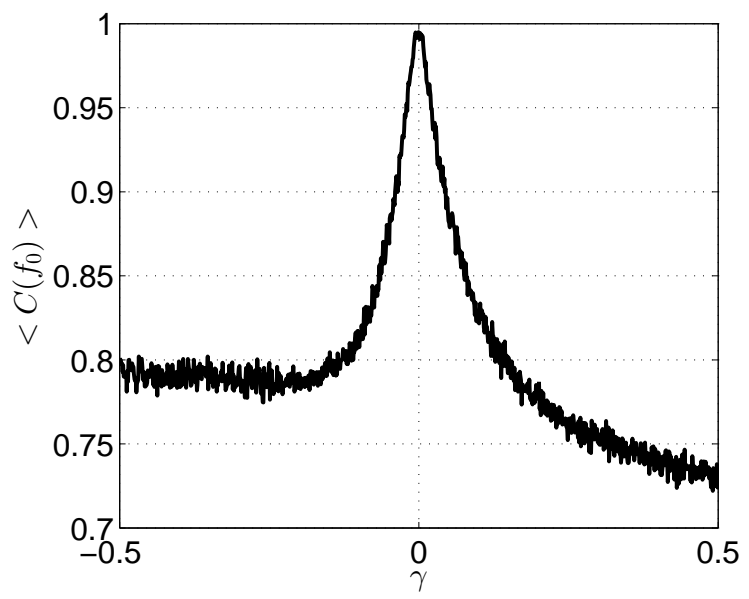

Figure 8: Spatially averaged coherence at the dominant frequency $f_{0}$ for the reconstruction of the analytical wavefield conducted with a wavenumber modified by Equation 13

correctly estimated within $\pm 5 \%$ hence resulting in a drop of the reconstruction coherence of less than $10 \%$ only, according to Figure 8.

\subsection{D experimental results}

The reconstruction process is applied to a wavefield measured experimentally on a quasi isotropic glass fiber specimen with the setup depicted in Figure 9. A Scanning Laser Doppler Vibrometer (SLDV) is employed for wavefield detection over a refined grid of points. A subset of the measurements is used for the problem formulation, while the entire set of measurements is retained for evaluation of the accuracy of the reconstruction. The wavefield is generated by a PZT disc bonded to the plate, and excited by a 4-cycle $300 \mathrm{kHz}$ sine burst. The specimen is a 8 layer glass fiber composite plate of dimension $600 \times 600 \times 1.6 \mathrm{~mm}$ and layup $[0 / 90 /+45 /-45]^{\circ}$. The specimen contains an artificial delamination created by inserting a Teflon disk of $25.4 \mathrm{~mm}$ in diameter between the second and the third ply. The PZT transducer is located approximately $100 \mathrm{~mm}$ away from the center of the delamination, and is outside 
the scanned region.

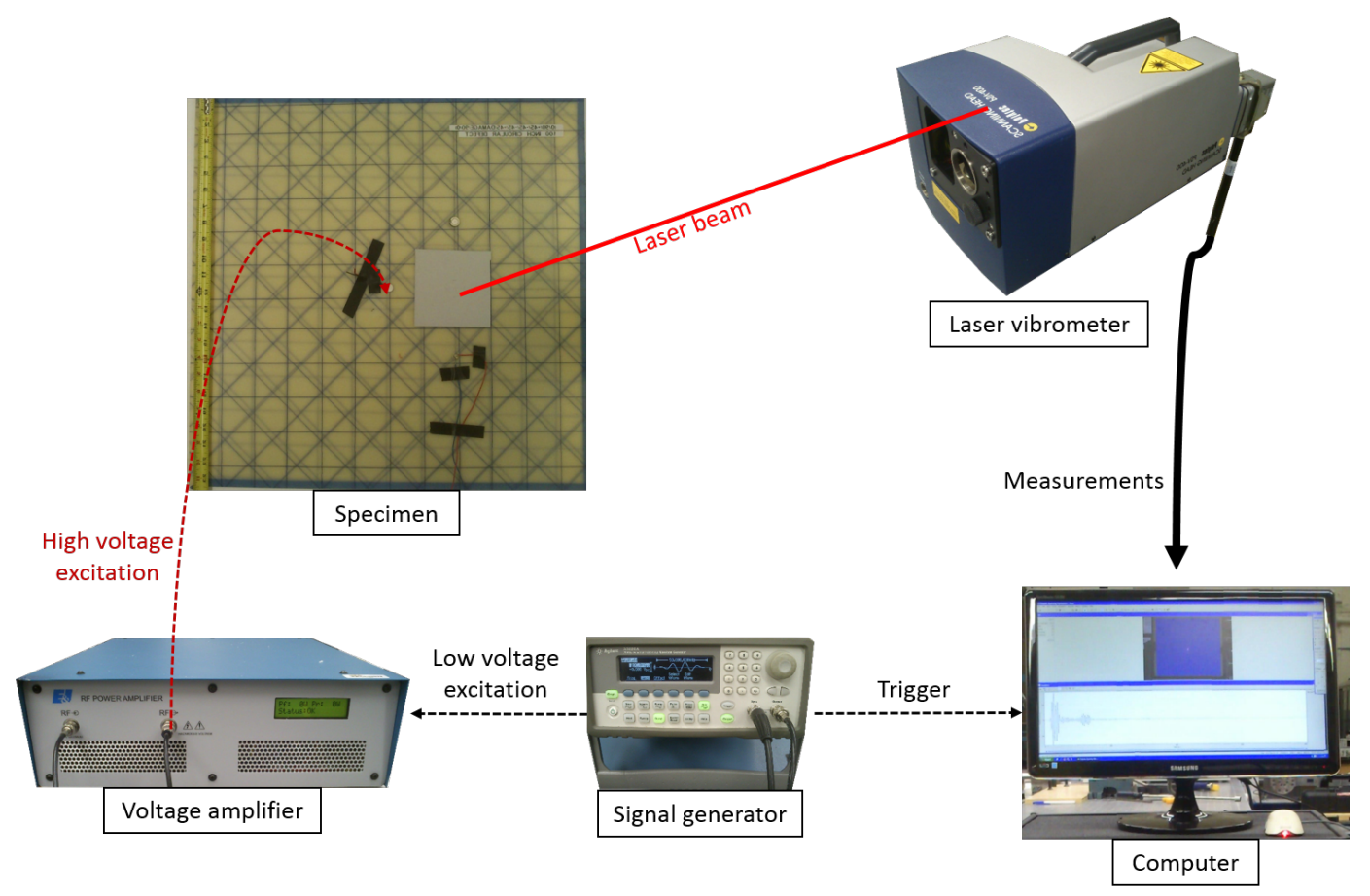

Figure 9: Experimental setup used to measure guided wavefields

The dispersion relation of the specimen are computed by SAFE [38] and are the same used in section 4.1. The following material properties provided by the supplier were used to generate the dispersion curves with SAFE: $E_{1}=47.8 \mathrm{GPa}, E_{2}=E_{3}=13.9 \mathrm{GPa}, G_{23}=5.2 \mathrm{GPa}$, $G_{12}=G_{31}=5.9 \mathrm{GPa}, \nu_{23}=0.3$ and $\nu_{12}=\nu_{31}=0.257$. Both $A 0$ and $S 0$ modes are generated and propagate in the plate in the frequency range of interest, which is $160-430 \mathrm{kHz}$. The corresponding minimum wavelength to be resolved is $\lambda_{\min }=2.9 \mathrm{~mm}$, which requires spatial sampling of the $111 \times 106 \mathrm{~mm}$ measurement region with a grid of $M_{N y q}=77 \times 74=5698$ points. The snapshot of the propagating wavefield in Figure 10 shows the presence of the two modes as well as the interaction with the delamination which scatters the incident waves and 
causes mode conversion.

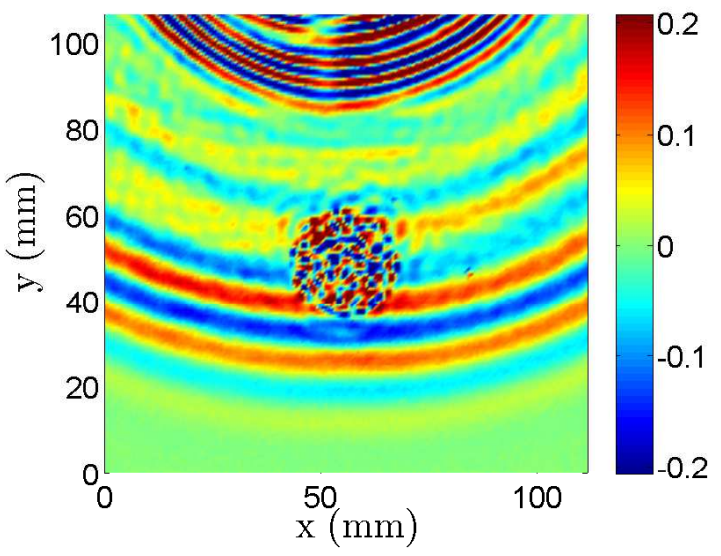

Figure 10: Snapshot of the measured wavefield at $t=50 \mu$ s showing propagation of two modes and interaction with the delamination.

The PZT transducer is located outside the measurement region, which requires a grid of potential sources to be defined over a $180 \times 106 \mathrm{~mm}$ area. This ensures that the main provenance of the wavefronts can be determined from the CS problem solution. A representation of the distributions of the potential sources and the measurements and the regions in which they are defined is shown in Figure 11. In general, the grid of potential sources should include all the wave interacting features of the structure. In this case, the acquisition time is limited to avoid the inclusion of edge reflections. Thus, the need to add the boundaries of the specimen as potential sources is avoided.

The $l 1$-minimization is conducted for $M=2000$ measurements $(C R=65 \%)$. The resulting amplitude of source excitation normalized to unity is shown in Figures $12 \mathrm{~b}$ and 12a, respectively for the $A 0$ and $S 0$ mode. In both figures, two clusters of active sources are visible in correspondence to the location of the PZT at $y=150 \mathrm{~mm}$, as well as in an area that outlines the shape of the delamination. The sparse representation of the PZT is a line approximately 


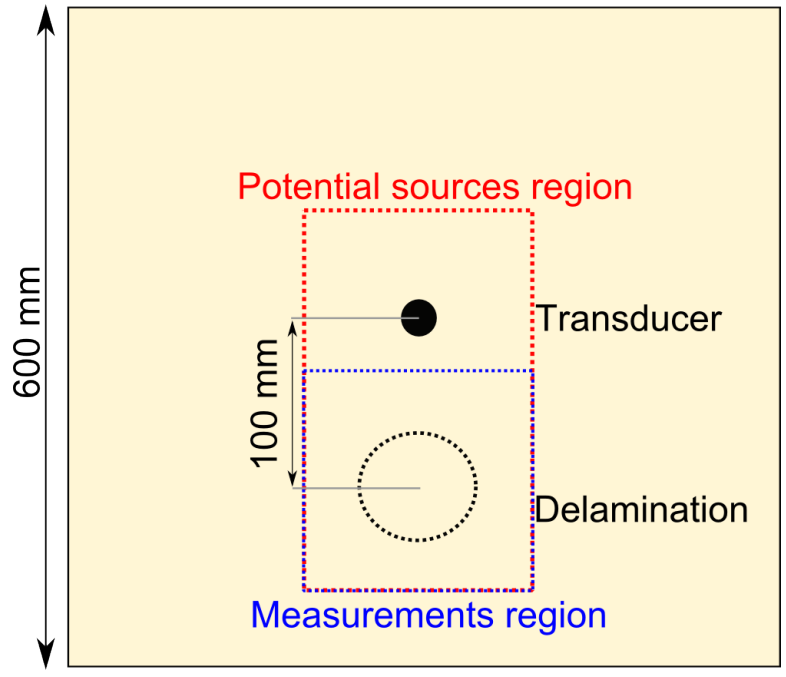

(a)

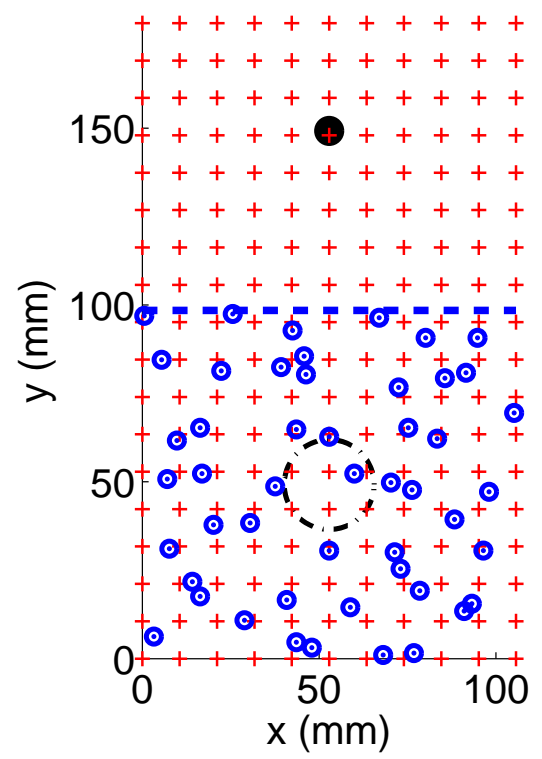

(b)

Figure 11: (a) Not to scale representation of the specimen. The red dashed lines represent the region of the potential sources, i.e. the region including all the origins of the wavefronts. The region in which the measurements are extracted and the wavefield is reconstructed is represented by the blue dashed box and (b) Representation to scale of the regions of interest, blue circles: Measurements following a jittered distribution and red crosses: Regular distribution of potential sources in the region including the transducer.

$15 \mathrm{~mm}$ long while the actual transducer is a $12 \mathrm{~mm}$ diameter disk. In this case, the sparse representation of the PZT is neither a circle nor a single point because the measurements are only taken in the south direction with respect to the transducer, so that only the wavepackets resulting of the elongation of the transducer along the y-axis are measured. It was found in other reconstructions (not presented in this paper) that selecting measurements in all the directions with respect to the transducer results in a circular cluster of active sources. Two main differences between Figure $12 \mathrm{~b}$ and 12a are observed. First, the location of the PZT 
transducer is sharply identified for the $S 0$, while the identification for $A 0$ appears more noisy. This is attributed to a mismatch observed upon measurement between actual wavenumbers and those numerically predicted and used in the basis functions. Such mismatch is approximately $2 \%$ for the $A 0$ mode, while it is less than $1 \%$ for $S 0$ mode. The lower definition of the $A 0$ source results from several active sources being identified by the $l 1$ minimization, which compensates for the mismatch in wavenumber estimations. Secondly, the excitation amplitude of the active sources in the delaminated area appears to be much greater for the $A 0$ mode than for the $S 0$. This is due to the fact the $A 0$ wavenumber is greater than the $S 0$ one, and better matches the wavenumber observed within the delaminated region. In fact, the employed basis only includes the two modal components for the undamaged plate, and has therefore limited ability to fully represent regions of different wavenumber content. In this case, as the wavenumber of the standing wave in the delaminated area is not present in the dictionary matrix $\boldsymbol{H}(f)$, there exist no sparse representation of the delamination, hence the delamination is represented by a full circle of active sources instead of a ring and the wavepackets are not successfully reconstructed in the delaminated region.

The estimated vector $\boldsymbol{v}(f)$ is then used for wavefield reconstruction. The result is shown in Figure 13 in terms of wavefield snapshot at a given time instant $(t=50 \mu \mathrm{s})$. The reconstructed wavefield matches quite well the measured one, with the exception of the delaminated region, where a lower wavenumber is observed. This is expected given the limitation of the basis used, and its corresponding limited ability to resolve pristine regions characterized by high wavenumber content. Furthermore, the wavepackets reflected from the defect are not correctly reconstructed because the immediate vicinity of the delaminated area is dominated by the superposition of the contributions from the cluster of sources constituting the non-sparse representation of the delamination. To increase the quality of the reflected wavepackets, the ratio between the number of measurements in the vicinity of the defect versus the number of 


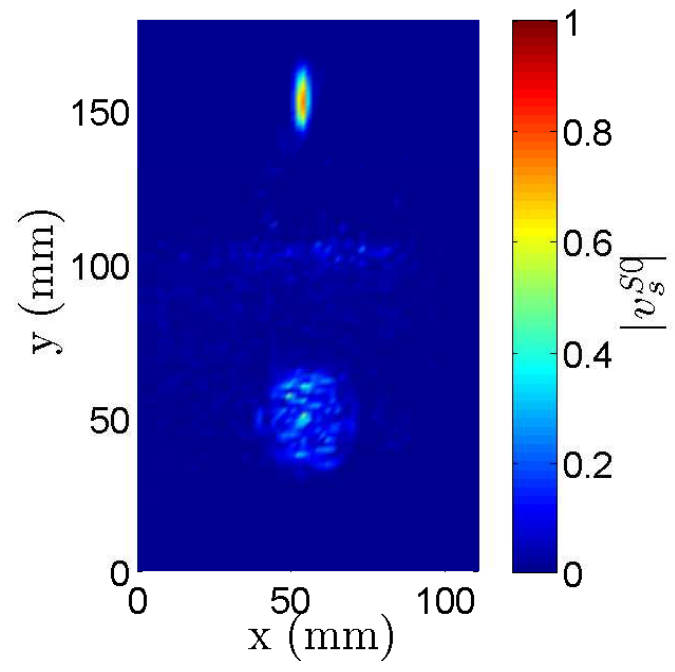

(a)

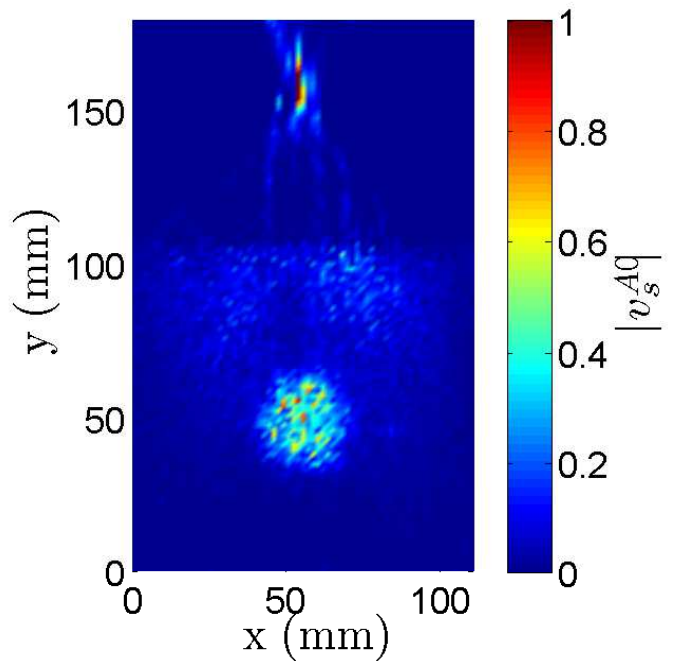

(b)

Figure 12: Active sources estimation averaged in frequency (Equation (11)) with $M=2000$ measurements: (a) $S 0$ mode and (b) $A 0$ mode.

measurements inside the defect would have to be increased.

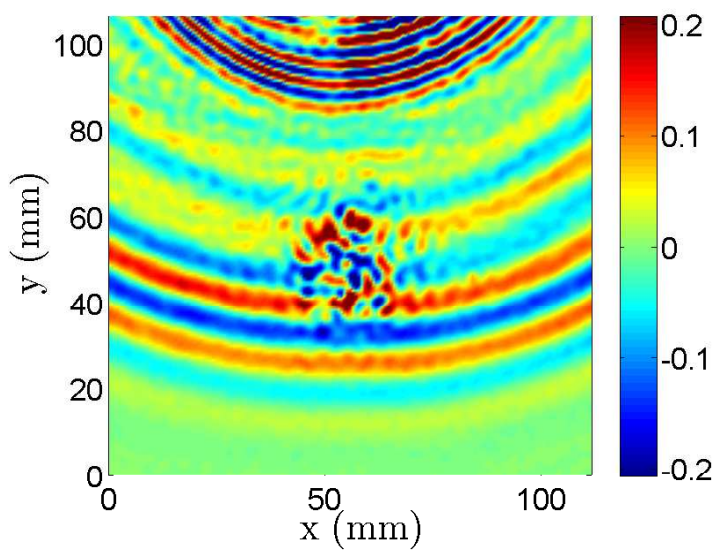

Figure 13: Snapshot of the reconstructed wavefield at $t=50 \mu$ s obtained using $M=2000$ measurements. 


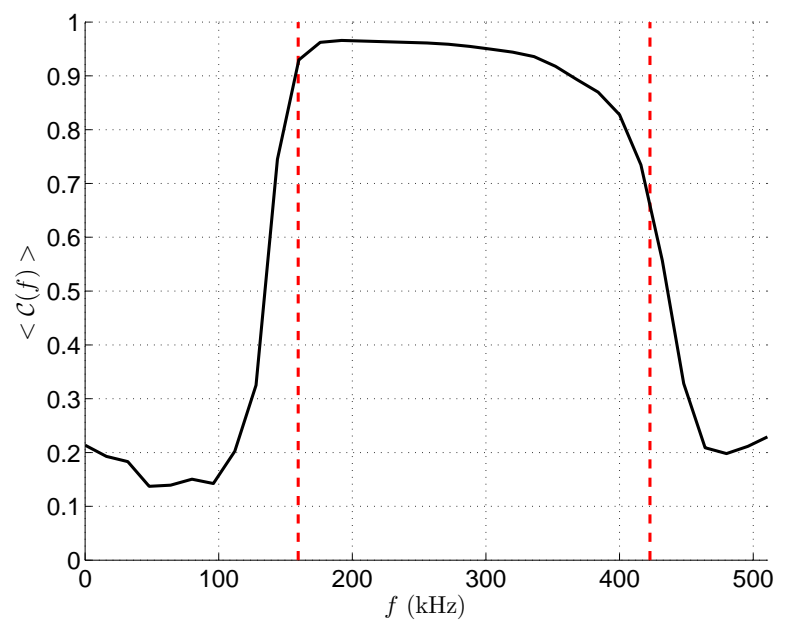

Figure 14: Spatially averaged coherence between true wavefield and wavefield reconstructed from $M=2000$. The red dashed lines represent the limit of the reconstruction

The coherence of the reconstruction is again used to quantify the quality of the reconstruction process. The spatially averaged coherence as a function of frequency is shown in Figure 14, where it can be seen that the coherence of the reconstruction is close to one within the limits of the reconstruction represented by the red dashed lines. Furthermore, the spatially averaged coherence at the excitation frequency $(300 \mathrm{kHz})$ as a function of number of measurements $M$ is used as a metric for the selection of $M=2000$ in the results presented above. The corresponding plot in Figure 15a shows that the number of measurements $M$ required for a coherence $\mathcal{C}>0.9$ is $M=636$, corresponding to a compression ration $C R=89 \%$. Thus, the proposed sparse reconstruction technique is able to reconstruct this wavefield with $\mathcal{C}>0.9$ from only $1 / 10^{\text {th }}$ the number of measurements required by Nyquist theorem, or only one measurement every 5 wavelengths. For reference, the coherence at the excitation frequency with $M=2000$ is $\mathcal{C}=0.951$. The fact that the coherence does not reach one can be explained by the non-modeled standing wave, which limits the ability to resolve the wavefield in the 
delaminated region which occupies $17 \%$ of the area of interest. This is illustrated by mapping the coherence over the measurement region at the dominant frequency for $M=2000$ (Figure 15b). The plot shows a value of the coherence close to one in the pristine area, while the value of the coherence is very low in the delaminated region.

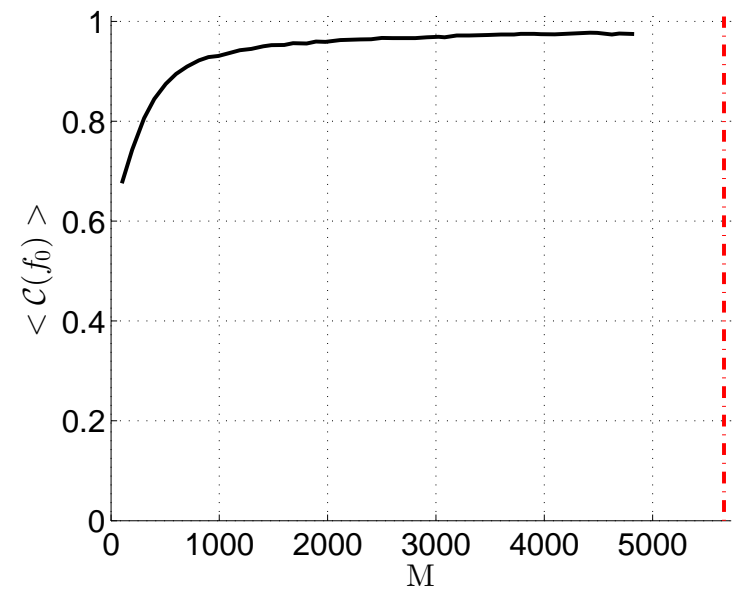

(a)

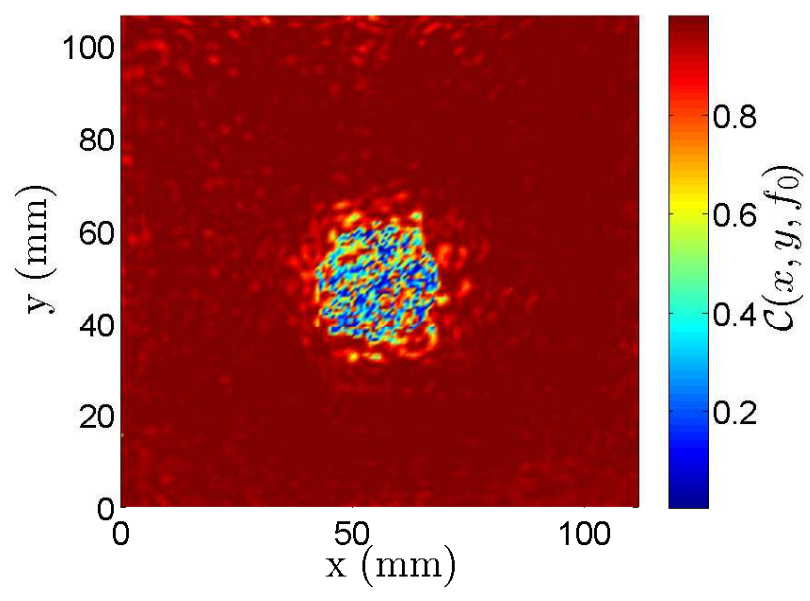

(b)

Figure 15: (a) Spatially averaged coherence at $300 \mathrm{kHz}$ versus number of measurements $M$; vertical red dashed line indicates Nyqvist sampling requirements. (b) Map of spatial variation of coherence at $300 \mathrm{kHz}$ and for $M=2000$. Reduction within delaminated region are due to limited wavenumber content in the basis.

\subsection{Comparison with other techniques}

As similar techniques have been recently developed by other research groups, it is necessary to compare the present technique to the existing ones. First, the technique developed in [20] achieves compression ratios up to $90 \%$ with what appears as comparable results quality. The main difference between this technique and the one presented in this paper is the choice of the basis functions to create the sparsifying basis, as explained in the introduction. Another comparable technique presented in [23] exploits a drastically different approach as the 
dispersion relations of the specimen are first learned from a small set of measurements and then used as basis functions for the reconstruction. To the best of our knowledge, this latest technique was only applied to reconstruct pristine wavefields. Overall, at the current stage of development, the presented reconstruction technique provides results and compression rates that are comparable to those recently presented $[20,23]$ that are obtained with a slightly different methodology.

\section{Conclusion}

A process for sparse reconstruction of a wavefield of $P$ pixels from $M$ measurements $(M<$ $P)$, is developed using Compressed Sensing. The process is validated through its application to a one-dimensional analytical wavefield and then applied to a two-dimensional experimental wavefield in a composite panel with a delamination. Compression ratios of the order of $90 \%$ with respect to Nyquist sampling criterion are demonstrated. The results presented suggest potential 10-fold reductions in the number of measurements required to measure a wavefield. In addition, the process accurately locates and quantifies the defects, and may separate modal components. Future work will investigate the increase in accuracy resulting from a richer basis, potentially to include wavenumbers corresponding to potential delamination scenarios that may be encountered in a layered structure. Moreover, the capability of the method to separate different modes will be further explored in order to improve damage identification resulting from mode conversions.

\section{Acknowledgment}

The work is funded by a collaborative agreement (NRANNH11ZEA001N VSST1) between NASA LaRC and Georgia Tech and the Strategic University Partnership between Boeing and 
Georgia Tech. The authors would also like to thank Joel Harley from the University of Utah, for the very useful discussions on the topic.

\section{References}

[1] Zhongqing Su, Lin Ye, and Ye Lu. Guided lamb waves for identification of damage in composite structures: A review. Journal of sound and vibration, 295(3):753-780, 2006.

[2] Jennifer E Michaels. Detection, localization and characterization of damage in plates with an in situ array of spatially distributed ultrasonic sensors. Smart Materials and Structures, 17(3), 2008.

[3] Seth S Kessler, S Mark Spearing, and Constantinos Soutis. Damage detection in composite materials using lamb wave methods. Smart Materials and Structures, 11(2):269, 2002.

[4] Victor Giurgiutiu. Lamb wave generation with piezoelectric wafer active sensors for structural health monitoring. Smart Structures and Materials 2003, 5056:111-122, 2003.

[5] Thomas Clarke, Peter Cawley, Paul David Wilcox, and Anthony John Croxford. Evaluation of the damage detection capability of a sparse-array guided-wave shm system applied to a complex structure under varying thermal conditions. Ultrasonics, Ferroelectrics and Frequency Control, IEEE Transactions on, 56(12):2666-2678, 2009.

[6] James Thomas Ayers. Structural damage diagnostics via wave propagation-based filtering techniques. PhD thesis, Georgia Institute of Technology, 2010.

[7] MD Rogge and CAC Leckey. Local guided wavefield analysis for characterization of delaminations in composites. In REVIEW OF PROGRESS IN QUANTITATIVE NON- 
DESTRUCTIVE EVALUATION: VOLUME 32, volume 1511, pages 963-970. AIP Publishing, 2013.

[8] H Sohn, D Dutta, JY Yang, M DeSimio, S Olson, and E Swenson. Automated detection of delamination and disbond from wavefield images obtained using a scanning laser vibrometer. Smart Materials and Structures, 20(4):045017, 2011.

[9] Thomas E Michaels, Jennifer E Michaels, and Massimo Ruzzene. Frequency-wavenumber domain analysis of guided wavefields. Ultrasonics, 51(4):452-466, 2011.

[10] WJ Staszewski, BC Lee, L Mallet, and F Scarpa. Structural health monitoring using scanning laser vibrometry: I. lamb wave sensing. Smart Materials and Structures, $13(2): 251,2004$.

[11] Olivier Mesnil, Cara AC Leckey, and Massimo Ruzzene. Instantaneous and local wavenumber estimations for damage quantification in composites. Structural Health Monitoring, 2014.

[12] E Cupido, S Morel, and D Smith. Multipoint laser doppler vibrometer for transient analysis. Proceedings of IMAC XXI, Orlando, USA, 2003.

[13] Yu Fu, Min Guo, and Poh Boon Phua. Multipoint laser doppler vibrometry with single detector: principles, implementations, and signal analyses. Applied optics, 50(10):1280$1288,2011$.

[14] Eric B. Flynn. Embedded mutli-tone ultrasonic excitation and continuousscanning laser doppler vibrometry for rapid and remote imaging of structural defects. In 7th European Workshop on Structural Health Monitoring, 2014. 
[15] Emmanuel J Candes. Compressive sampling. In Proceedings oh the International Congress of Mathematicians: Madrid, August 22-30, 2006: invited lectures, pages 1433$1452,2006$.

[16] D.L. Donoho. Compressed sensing. IEEE Trans. Inform. Theory, 52:1289-1306, 2006.

[17] Marco F. Duarte, Mark A. Davenport, Dharmpal Takhar, Jason N. Laska, Ting Sun, Kevin F. Kelly, and Richard G. Baraniuk. Single-pixel imaging via compressive sampling. IEEE Signal Processing Magazine, 25(2):83, 2008.

[18] Michael Lustig, David L Donoho, Juan M Santos, and John M Pauly. Compressed sensing mri. Signal Processing Magazine, IEEE, 25(2):72-82, 2008.

[19] Tommaso Di Ianni, Alessandro Perelli, Luca De Marchi, and Alessandro Marzani. Compressive sensing for full wavefield image recovery in structural monitoring applications. In 7th European Workshop on Structural Health Monitoring, 2014.

[20] Tommaso Di Ianni, Luca De Marchi, Alessandro Perelli, and Alessandro Marzani. Compressive sensing of full wave field data for structural health monitoring applications. Ultrasonics, Ferroelectrics, and Frequency Control, IEEE Transactions on, 62(7):1373$1383,2015$.

[21] Joel B Harley and Jose MF Moura. Sparse recovery of the multimodal and dispersive characteristics of lamb waves. The Journal of the Acoustical Society of America, 133:2732-2745, 2013.

[22] Joel B. Harley and Jose M. F. Moura. Data-driven matched field processing for lamb wave structural health monitoring. The Journal of the Acoustical Society of America, 135(3):1231-1244, Mar 2014. 
[23] Joel B. Harley. Characterizing and predicting guided wave eigenmode behavior through sparse representations. IEEE, 2015.

[24] Stéphane G Mallat and Zhifeng Zhang. Matching pursuits with time-frequency dictionaries. Signal Processing, IEEE Transactions on, 41(12):3397-3415, 1993.

[25] Yagyensh Chandra Pati, Ramin Rezaiifar, and PS Krishnaprasad. Orthogonal matching pursuit: Recursive function approximation with applications to wavelet decomposition. In Signals, Systems and Computers, 1993. 1993 Conference Record of The Twenty-Seventh Asilomar Conference on, pages 40-44. IEEE, 1993.

[26] Shaobing Chen and David Donoho. Basis pursuit. In Signals, Systems and Computers, 1994. 1994 Conference Record of the Twenty-Eighth Asilomar Conference on, volume 1, pages 41-44. IEEE, 1994.

[27] Donoho, Chen, and Saunders. Atomic decomposition by basis pursuit. SIAM J. Sci. Comput, 20:33-61, 1998.

[28] Chengbo Li. An efficient algorithm for total variation regularization with applications to the single pixel camera and compressive sensing. $\mathrm{PhD}$ thesis, Citeseer, 2009.

[29] Leonid I Rudin, Stanley Osher, and Emad Fatemi. Nonlinear total variation based noise removal algorithms. Physica D: Nonlinear Phenomena, 60(1):259-268, 1992.

[30] Emmanuel J Candes, Yonina C Eldar, Deanna Needell, and Paige Randall. Compressed sensing with coherent and redundant dictionaries. Applied and Computational Harmonic Analysis, 31(1):59-73, 2011.

[31] Afonso S Bandeira, Edgar Dobriban, Dustin G Mixon, and William F Sawin. Certifying the restricted isometry property is hard. arXiv preprint arXiv:1204.1580, 2012. 
[32] Sara Cohen. Compressive sensing with highly coherent dictionaries. Undergraduate Senior Thesis, University of California, Davis, 2012.

[33] Emmanuel J Candès, Yaniv Plan, et al. Near-ideal model selection by 11 minimization. The Annals of Statistics, 37(5A):2145-2177, 2009.

[34] Yair Weiss, Hyun Sung Chang, and William T Freeman. Learning compressed sensing. In Snowbird Learning Workshop, Allerton, CA. Citeseer, Citeseer, 2007.

[35] E. van den Berg and M. P. Friedlander. Probing the pareto frontier for basis pursuit solutions. SIAM Journal on Scientific Computing, 31(2):890-912, 2008.

[36] E. van den Berg and M. P. Friedlander. SPGL1: A solver for large-scale sparse reconstruction, June 2007. http://www.cs.ubc.ca/labs/scl/spgl1.

[37] James S Hall and Jennifer E Michaels. Model-based parameter estimation for characterizing wave propagation in a homogeneous medium. Inverse Problems, 27(3):035002, 2011.

[38] Ivan Bartoli, Alessandro Marzani, Francesco Lanza di Scalea, and Erasmo Viola. Modeling wave propagation in damped waveguides of arbitrary cross-section. Journal of Sound and Vibration, 295(3):685-707, 2006.

[39] Gilles Hennenfent and Felix J Herrmann. Simply denoise: wavefield reconstruction via jittered undersampling. Geophysics, 73(3):V19-V28, 2008.

[40] Ross Levine. Ultrasonic Guided Wave Imaging via Sparse Reconstruction. PhD thesis, Georgia Institute of Technology, 2014. 
[41] Olivier Mesnil, Hao Yan, Massimo Ruzzene, Kamran Paynabar, and Jianjun Shi. Fast wavenumber measurement for accurate and automatic location and quantification of defect in composite. Manuscript submitted for publication.

[42] Peter D Juarez and Cara AC Leckey. Multi-frequency local wavenumber analysis and ply correlation of delamination damage. Ultrasonics, 2015. 